\title{
A cluster of specified microRNAs in peripheral blood as biomarkers for metastatic non-small-cell lung cancer by stem-loop RT-PCR
}

\author{
Qingfeng Lin · Weidong Mao • Yongqian Shu • \\ Feng Lin - Shaoping Liu - Hua Shen · \\ Wen Gao $\cdot$ Suqing $\mathbf{~ L i} \cdot$ Dong Shen
}

Received: 7 May 2011/Accepted: 12 September 2011/Published online: 19 October 2011

(C) The Author(s) 2011. This article is published with open access at Springerlink.com

\begin{abstract}
Purpose To investigate the levels for some specified microRNAs in human's peripheral blood so as to determine whether they can serve as biomarkers for metastatic nonsmall-cell lung cancer.

Methods Use a quantitative stem-loop RT-PCR method to examine the serum levels for certain microRNAs including has-miR-125a-5p, has-miR-126, has-miR-183, has-miR-200, has-miR-221, and has-miR-222 from the patients with Stage IV, Stage I/II non-small-cell lung cancer and the controls.

Results There was statistical difference in the serum levels for hsa-miR-126, hsa-miR-183, and hsa-miR-222 between the controls and the Stage IV patients, but not for has-miR125a-5p, has-miR-200 and has-miR-221. It also showed statistical difference for hsa-miR-126 and hsa-miR-183 between the Stage I/II patients and Stage IV patients, but not between the controls and Stage I/II patients.

Conclusion Hsa-miR-126 and hsa-miR-183 may serve as potential serum biomarkers for metastatic non-small-cell lung cancer.
\end{abstract}

Keywords microRNAs · Non-small-cell lung cancer · Stem-loop RT-PCR · Biomarker

Q. Lin · W. Mao $(\varangle) \cdot$ F. Lin · S. Liu · D. Shen

Oncology Department, Affiliated Jiangyin Hospital of Southeast

University Medical College, Jiangyin, Jiangsu, China

e-mail: wxjymwd@126.com

Y. Shu $\cdot$ H. Shen $\cdot$ W. Gao

Oncology Department, The First Affiliated Hospital of Nanjing

Medical University, Nanjing, Jiangsu, China

S. Li

Department of Oncology Surgery, Affiliated Nanjing First Hospital, Nanjing Medical University, Nanjing, Jiangsu, China

\section{Introduction}

Lung cancer, predominantly non-small-cell lung cancer (NSCLC), is the most common cause of cancer deaths worldwide (Jemal et al. 2006). NSCLC includes two predominant subtypes, adenocarcinoma and squamous cell carcinoma, which comprise 40 and $25 \%$, respectively (Dutt et al. 2011). Unfortunately, local and/or distant metastases have developed in up to $75 \%$ of the lung cancer patients when clinically diagnosed (Coello et al. 2004). So it becomes important to find a reliable and feasible way to discriminate the metastases.

Invasion of a primary tumor into surrounding tissues followed by dissemination and growth of secondary malignancies (metastasis) require a concerted series of events including dissociation of primary tumor cells from the primary neoplasia, entry into the circulatory system, and colonization at distant sites. The series of steps needed for metastatic spread involve changes in the expression of many genes (Du and Pertsemlidis 2010). Investigations of microRNAs (miRNAs) regulation of these steps have identified several specific miRNAs that either promote or inhibit metastatic potential. MiR-125a-5p was reported to inhibit migration and invasion of lung cancer cells by regulating the expression of several downstream genes of epidermal growth factor receptor signaling (Wang et al. 2009a). Overexpression of miR-126 in a lung cancer cell line resulted in a decrease in Crk protein that is a member of a family of adaptor proteins involved in intracellular signal pathways altering cell adhesion, proliferation, and migration (Crawford et al. 2008). Expression level of miR-183 was demonstrated to reversely correlate with the metastatic potential of lung cancer cells by targeting VIL2-coding protein ezrin and regulating the expression of other genes involved in migration and invasion (Wang et al. 2008). MiR-200 inhibits lung 
adenocarcinoma cell invasion and metastasis by targeting Flt1/VEGFR1 in a mouse model (Roybal et al. 2011). MiR$221 \& 222$ enhance cellular migration through the activation of the AKT pathway (Garofalo et al. 2009).

Moreover, it has been demonstrated that serum/plasma miRNAs derived from various tissues/organs are stable and resistant to nuclease digestion. Expression levels of miRNAs in blood have been found to be reproducible and indicative of the disease status (Chen et al. 2008). Thus, specific signatures of blood miRNA could be obtained from total blood samples and can be used in the identification of biomarkers for diagnosis, prognosis, or even the etiology of a disease. In this study, using blood samples collected from the Stage IV NSCLC patients, the Stage I/II NSCLC patients, and healthy controls, we intended to find a cluster of miRNAs that can be used as new risk markers for the diagnosis of lung cancer metastasis.

\section{Materials and methods}

Controls and patients' samples

Peripheral blood was collected in EDTA anticoagulation tubes from 20 healthy controls, 30 NSCLC patients of Stage IV, and 32 patients of Stage I/II according to TNM stage of 2007. All the individuals enrolled gave their informed consent, and the Ethical and Scientific Committees of the hospital approved the study. None of the controls had previously been diagnosed with a malignancy. Among the controls, 10 were male and 10 female. And of the Stage IV patients, 13 cases were diagnosed with lung squamous cell carcinoma, while 17 cases adenocarcinoma. Of the Stage I/II patients, 20 cases were of Stage I and 12 of Stage II.

The blood was centrifuged at 2,000 rpm for $20 \mathrm{~min}$, within $4 \mathrm{~h}$ after the collection, and then the serum was separated from the blood immediately, frozen in the refrigerator, and stored at $-80^{\circ} \mathrm{C}$ until RNA isolation. During the sample storage, repeated freeze-thawing were avoided to ensure the quality of the samples.

Extraction of total RNA from the serum

The total RNA including small RNAs was extracted from $200 \mu \mathrm{L}$ of the serum using the Qiagen Cat 217,004 kit according to the manufacturer's instructions. RNA quality was assessed with the Thermo Scientific Nano Drop 1,000 spectrophotometer (Thermo Scientific).

RT-PCR for miRNA quantification

Firstly, we tested the serum levels of the specified miRNAs from the 20 controls and the 30 Stage IV NSCLC patients.
We modified a stem-loop quantitative RT-PCR method to quantify the expression of the miRNAs including miR125a-5p, miR-126, miR-183, miR-200, miR-221, miR-222, and U6 in the Light Cycler platform. Then the miRNAs that had statistical difference between the controls and Stage IV NSCLC patients were quantified from the serum of the Stage I/II NSCLC patients. Normalization was performed with the small nuclear RNA (snRNA) U6. This snRNA is a stably expressed reference gene suitable for use as normalizers. The sequences of the miRNAs and U6 were obtained from the miRbase (V16.0) on line, and the primers used in this study were designed by Sangon Biotech (Shanghai) Co., Ltd. with the software DNA Star 6.1.3. The final procedure of stem-loop RT-PCR includes the following two main steps.

Reverse transcription with miRNA-specific stem-loop primers

We used $2.5 \mu \mathrm{L}$ total RNA to perform reverse transcription of RNA with the Applied Biosystems ABI7900 according to the manufacturer's instructions. We added $0.5 \mu \mathrm{L}$ miRNA-specific primers and $2.5 \mu \mathrm{L}$ total RNA to incubate at $70^{\circ} \mathrm{C}$ for $10 \mathrm{~min}$ and then snap on ice for $3 \mathrm{~min}$. So the total RNA got denatured. Then we added $3.0 \mu \mathrm{L}$ of the mixture of the denatured total RNA and RT primers, 0.25 $\mu \mathrm{L}$ dNTP (10 mM each, Takara, D4030RA), $1.00 \mu \mathrm{L}$ RT buffer $(5 \times), 0.25 \mu \mathrm{L}$ RNase inhibitor $(40 \mathrm{U} / \mu \mathrm{L}$, Takara, $\mathrm{D} 2313 \mathrm{~A})$, and $0.5 \mu \mathrm{L}$ M-MLV $(200 \mathrm{U} / \mu \mathrm{L}$, Takara, D2639A), performed the RT reaction in a total volume of 5 $\mu \mathrm{L}$ at $42^{\circ} \mathrm{C}$ for $60 \mathrm{~min}$, then deactivated the enzymes at $70^{\circ} \mathrm{C}$ for $15 \mathrm{~min}$, hold at $4^{\circ} \mathrm{C}$, and finally we got the RT product. Meanwhile, the negative controls with no template (NTC) were included for the miRNAs.

\section{Quantification of the miRNAs and U6 expression} by RT-PCR

Real-time PCR was performed in a total volume of $20 \mu \mathrm{L}$ per reaction in triplicate. We placed $1 \mu \mathrm{L}$ of $\mathrm{RT}$ product described previously into a $19 \mu \mathrm{L}$ reaction mixture that contained 10.0 ul Platinum SYBR Green qPCR Super MixUDG (Invitrogen, 11733-038), $0.4 \mu \mathrm{L}$ ROX reference dye, $7.8 \mu \mathrm{L}$ diethylpyrocarbonate (DEPC)-treated $\mathrm{H}_{2} \mathrm{O}, 0.8 \mu \mathrm{L}$ primer mix of the forward primers and the reverse primers $(10 \mathrm{uM})$, and $1.0 \mu \mathrm{L}$ RT product. The cycling protocol consisted of an initial 2-min step at $50^{\circ} \mathrm{C}$ to clear the DNA residue of the previous time, a 2-min initial denaturation step at $95^{\circ} \mathrm{C}$, followed by 39 cycles of denaturation at $95^{\circ} \mathrm{C}$ for $15 \mathrm{~s}$, annealing and extension at $60^{\circ} \mathrm{C}$ for $30 \mathrm{~s}$, and then plate-reading of fluorescence signals, melt-curve analysis from 65 to $90^{\circ} \mathrm{C}$. Real-time PCR for U6 gene was performed in the same way, by using the corresponding primers. 
Statistical analysis

Careful normalization is essential for accurate quantification of miRNAs. We evaluated all the data with respect to the expression of the miRNAs described previously by normalizing to the expression of U6 and using the $2-\Delta \Delta \mathrm{Ct}$ method. We assessed the differentially expression of the miRNAs in the controls and the patients with different clinicopathological features using $t$ test. All the data were analyzed with SPSS software (version 11.0, SPSS), and $P<0.05$ was considered statistically significant.

\section{Results}

We firstly measured the expression levels for miR-125a-5p, miR-126, miR-183, miR-200, miR-221, miR-222, and U6 in serum samples from the 20 healthy controls and 30 Stage IV NSCLC patients, and then we chosed to examine the serum levels of miR-126, miR-183, miR-222, and U6 from 32 Stage I/II NSCLC patients according to the preceding results (primers for the miRNAs shown in Table 1). The sequence data have been submitted to the miRbase V16.0 under accession number MIMAT0000443, MIMAT00 00445, MIMAT0000261, MIMAT0000682/MIMAT00003 18/MIMAT0000617, MIMAT0000278, MIMAT0000279, and NR_004394.1 in turn. All the miRNAs listed above could be detected in all the serum from the patients and the controls by stem-loop RT-PCR.

Comparison of the serum miRNAs between the male and female controls

Although all of the miRNAs listed previously were reported to have relation to lung cancer metastasis, we demonstrate that they were present in the serum from the 20 healthy controls. To determine whether the miRNAs levels of the man and woman were different, we analyzed the serum levels for these miRNAs from the controls. And no statistical difference was observed for the expression of any miRNA listed previously between the serum of the male and female controls (Fig. 1, $P>0.05$ ).

Comparison of the serum miRNAs between the Stage IV NSCLC patients of adenocarcinoma and squamous cell cancer

Some studies have indicated that certain miRNAs can be used to discriminate different pathological types of lung cancer. However, we observed no statistical difference for serum levels of any miRNA listed above between the Stage IV NSCLC patients of adenocarcinoma and squamous cell carcinoma (Fig. 2, $P>0.05$ ).

Table 1 Primers for miR-125a-5p, miR-126, miR-183, miR-200, miR-221, and miR-222

\begin{tabular}{|c|c|c|c|}
\hline \multirow[t]{2}{*}{ microRNAs } & \multirow[t]{2}{*}{ Reverse-transcribed primers } & \multicolumn{2}{|l|}{ PCR primers } \\
\hline & & Forward & Reverse \\
\hline \multirow[t]{3}{*}{ has-miR-125a-5p } & GTCGTATCCAGTGCAGG & CGGCTCCCTG & CAGTGCAGGG \\
\hline & GTCCGAGGTATTCGCAC & AGACCCTTTA & TCCGAGGTATT \\
\hline & TGGATACGACTCACAG & & \\
\hline \multirow[t]{3}{*}{ has-miR-126 } & GTCGTATCCAGTGCAGG & GGCGCTCGTA & CAGTGCAGGG \\
\hline & GTCCGAGGTATTCGCACT & CCGTGAGTAA & TCCGAGGTATT \\
\hline & GGATACGACCGCATT & & \\
\hline \multirow[t]{3}{*}{ has-miR-183 } & GTCGTATCCAGTGCAGG & CGCGGTATGG & AGTGCAGGGT \\
\hline & GTCCGAGGTATTCGCAC & CACTGGTAGA & CCGAGGTATTC \\
\hline & TGGATACGACAGTGAA & & \\
\hline \multirow[t]{3}{*}{ has-miR-200 } & GTCGTATCCAGTGCAGG & CGCGGTAACAC & AGTGCAGGGT \\
\hline & GTCCGAGGTATTCGCAC & TGTCTGGTAAC & CCGAGGTATTC \\
\hline & TGGATACGACACATCG & & \\
\hline \multirow[t]{3}{*}{ has-miR-221 } & GTCGTATCCAGTGCAGG & CCGCGGAGC & CAGTGCAGGG \\
\hline & GTCCGAGGTATTCGCAC & TACATTGTCT & TCCGAGGTATT \\
\hline & TGGATACGACGAAACC & & \\
\hline \multirow[t]{3}{*}{ has-miR-222 } & GTCGTATCCAGTGCAGG & GCGCAGCTACA & CAGTGCAGGG \\
\hline & GTCCGAGGTATTCGC & TCTGGCTACT & TCCGAGGTATT \\
\hline & ACTGGATACGACACCCAG & & \\
\hline \multirow[t]{2}{*}{ U6 snRNA } & CGAATTTGCGTGTCATCCT & CTCGCTTCG & CGAATTTGC \\
\hline & & GCAGCACATA & GTGTCATCCT \\
\hline
\end{tabular}


Fig. 1 The serum levels of miR-125a-5p, miR-126, miR183, miR-200, miR-221, and miR-222 from the healthy controls were tested by stemloop RT-PCR. There was no statistical difference for the expression of any miRNA listed above between the serum samples of the male and female controls $(P>0.05)$
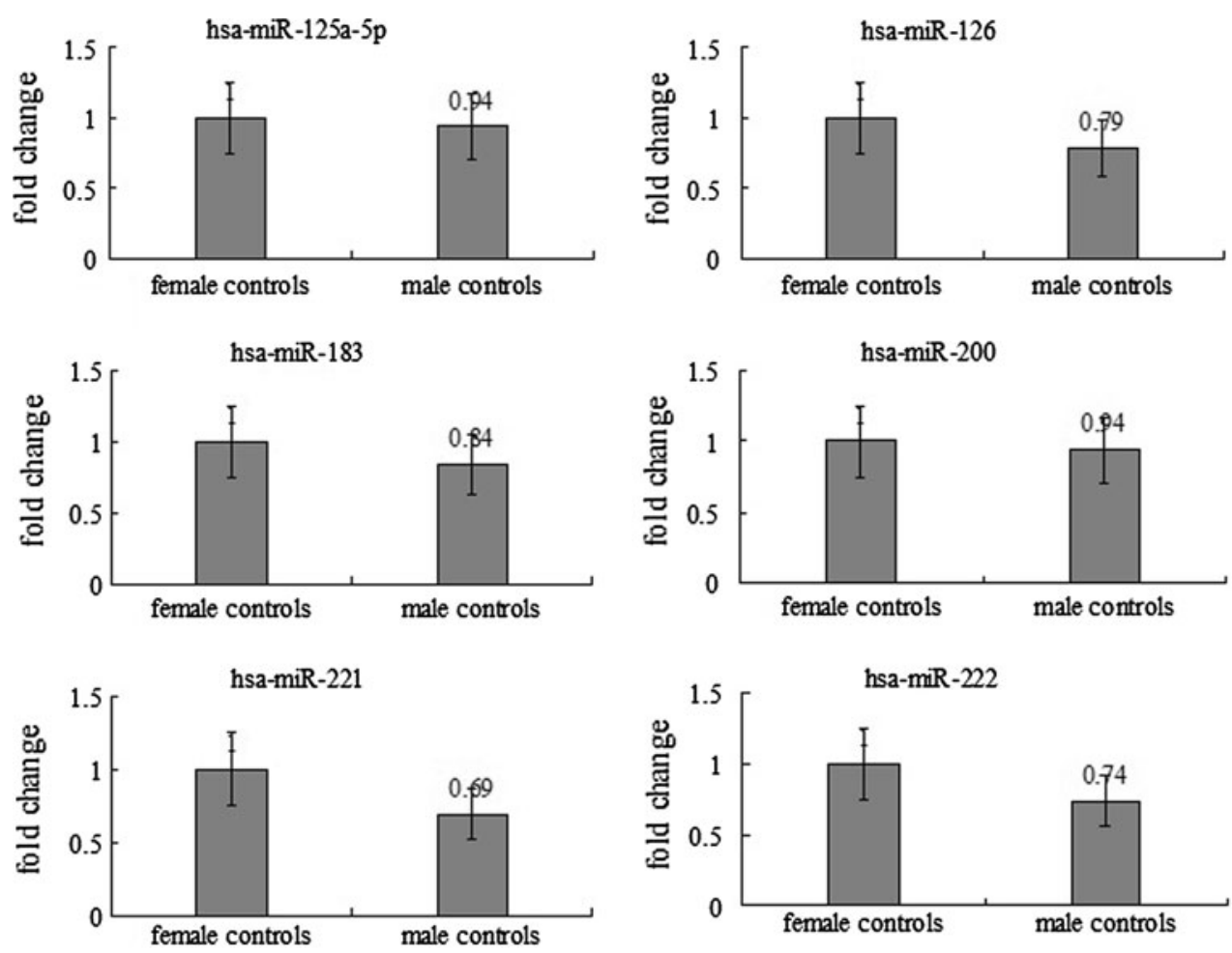

Fig. 2 The serum levels of miR-125a-5p, miR-126, miR183, miR-200, miR-221, and miR-222 from the Stage IV NSCLC patients were tested by stem-loop RT-PCR. There was no statistical difference for the expression of any miRNA listed above between the serum samples of the adenocarcinoma patients and squamous cell cancer patients $(P>0.05)$
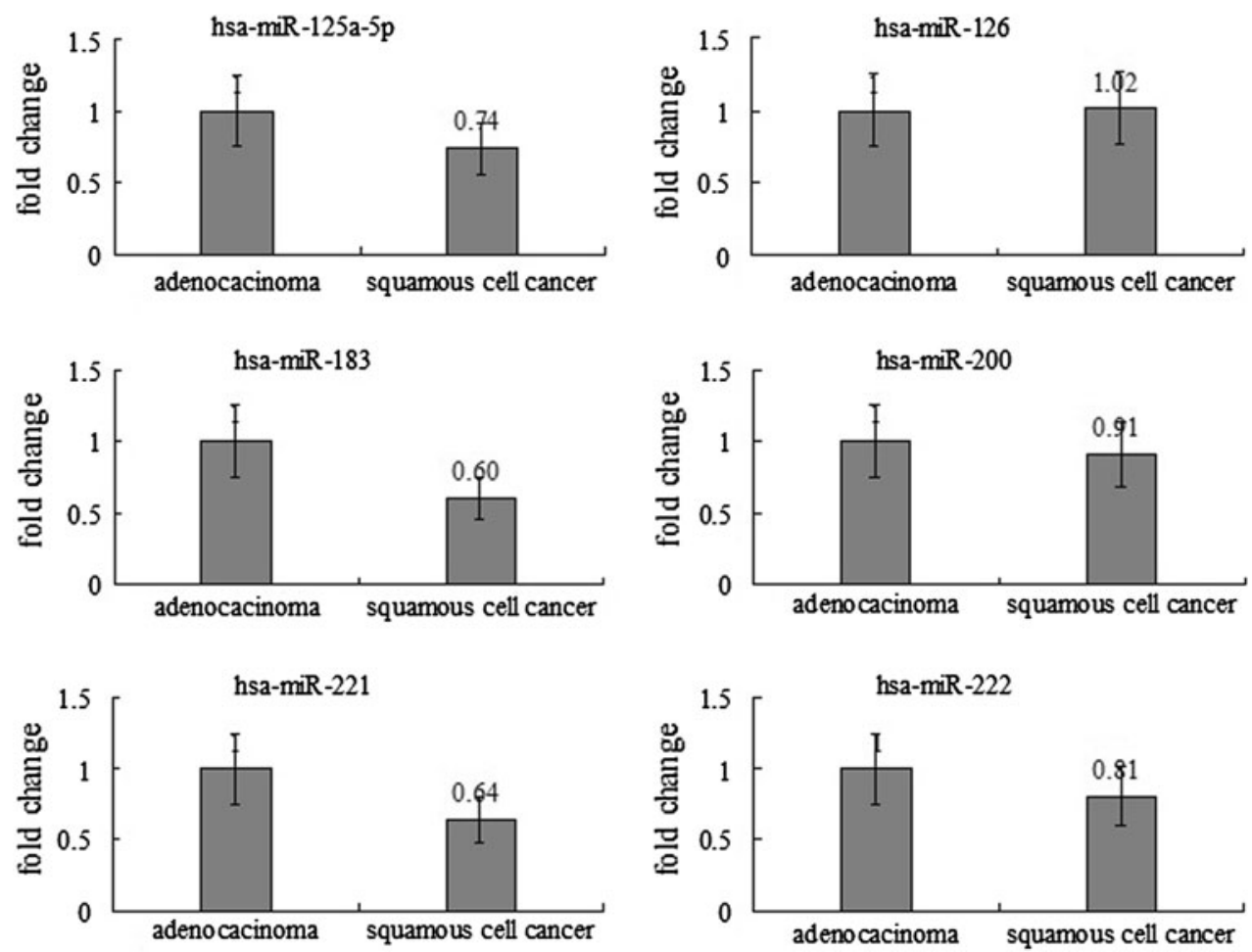

Comparison of the serum miRNAs between the controls and Stage IV NSCLC patients

Since these specified miRNAs have been reported to play various roles in the metastasis procedure of lung cancer in vitro, we attempted to study the expression levels of them from human's serum. As shown in Fig. 3, there was statistical difference for the levels of hsa-miR-126, hsa-miR183, and hsa-miR-222 between the serum of the Stage IV NSCLC patients and the controls $(P<0.05)$, but not for 
Fig. 3 The serum levels of miR-125a-5p, miR-126, miR183, miR-200, miR-221, and miR-222 from the controls and the Stage IV NSCLC patients were tested by stem-loop RTPCR. There was statistical difference for the levels of hsamiR-126, hsa-miR-183, and hsa-miR-222 between the serum of the Stage IV NSCLC patients and the controls $(P<0.05)$, but not for the levels of hsa-miR200, hsa-miR-125a-5p, and hsamiR-221 $(P>0.05)$
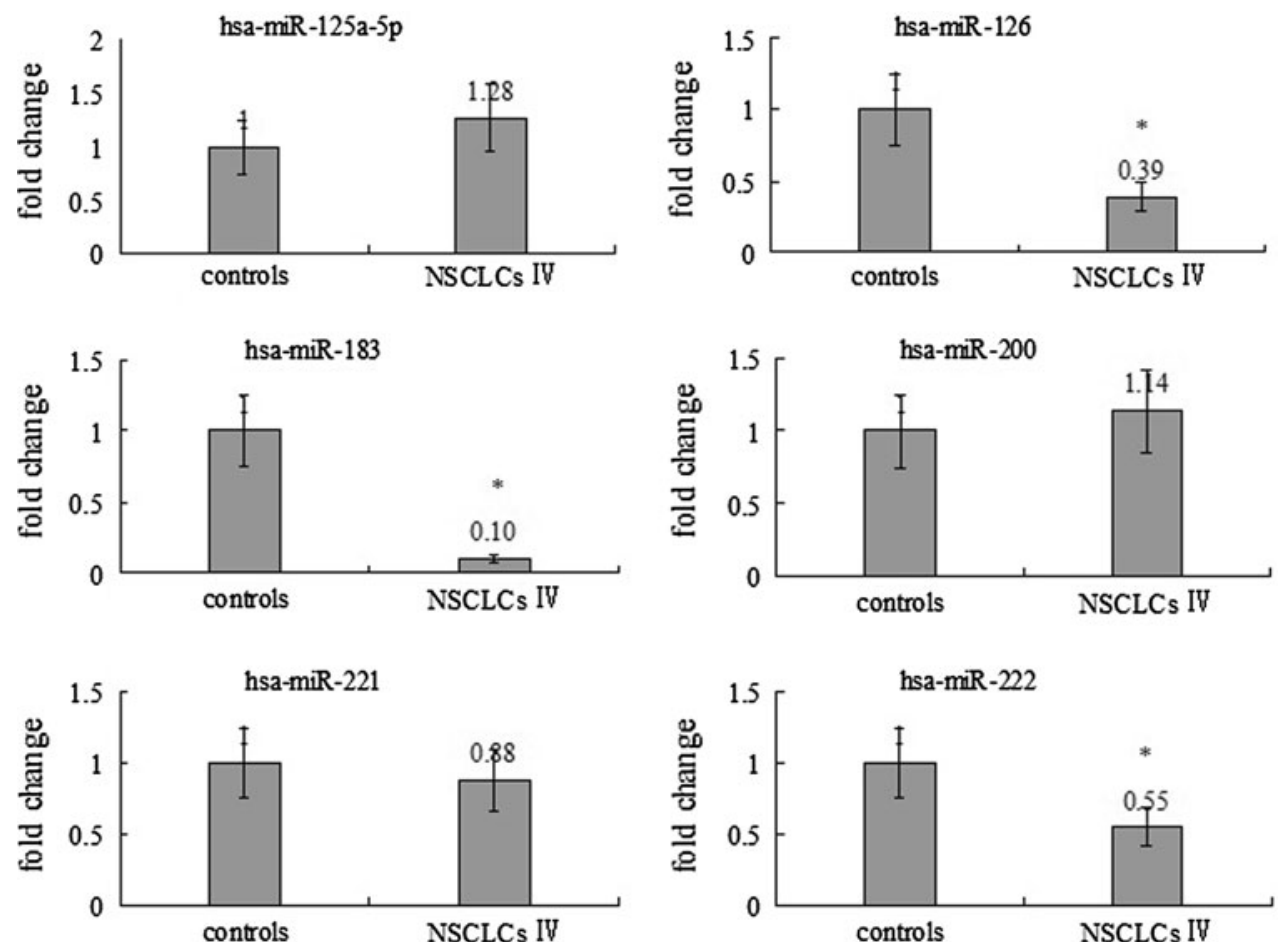

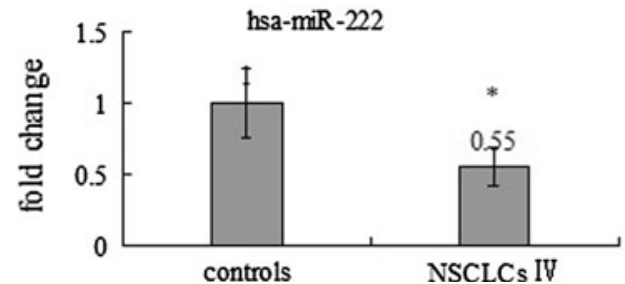

hsa-miR-126 the levels of hsa-miR-200, hsa-miR-125a-5p, and hsa-miR$221(P>0.05)$. It seemed that the serum levels of hsamiR-126, hsa-miR-183, and hsa-miR-222 may have closer relation to lung cancer metastases.

Comparison of the serum miRNAs between the controls and Stage I/II NSCLC patients, and between Stage I/II NSCLC patients and Stage IV NSCLC patients

Since the preceding results had shown that the serum levels of hsa-miR-126, hsa-miR-183, and hsa-miR-222 may be closely related to lung cancer metastases, we chose these three miRNAs for further investigation. We made further efforts to examine the serum levels for hsa-miR-126, hsamiR-183, and hsa-miR-222 from the Stage I/II NSCLC patients at exactly the same conditions as described previously, so that the data were credible and comparable. Then we analyzed the serum levels of these three miRNAs between the controls and Stage I/II NSCLC patients, and between the Stage I/II NSCLC patients and the Stage IV NSCLC patients. As a result, there was no statistical difference for hsa-miR-126, hsa-miR-183, and hsa-miR-222 between the controls and Stage I/II patients $(P>0.05)$. Meanwhile, it showed statistical difference for hsa-miR126 and hsa-miR-183 between the Stage I/II patients and Stage IV patients $(P<0.05)$, but not for hsa-miR-222 $(P>0.05)$ (Figs. 4, 5, and 6). The data indicated that serum levels for hsa-miR-126 and hsa-miR-183 may be truly related to the procedure of lung cancer metastases.

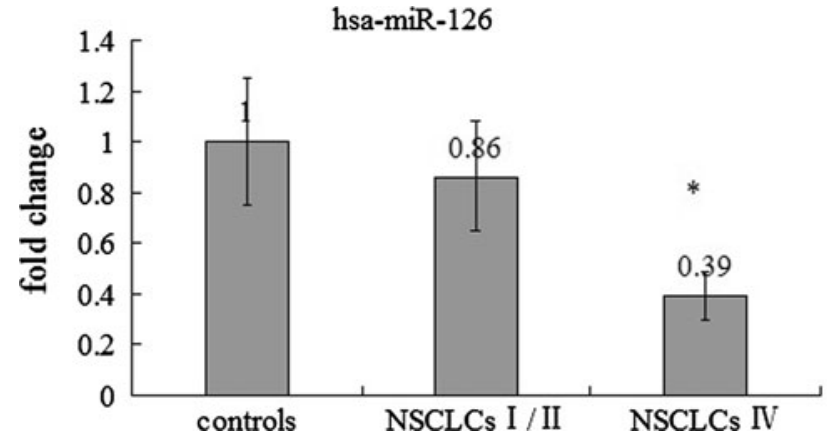

Fig. 4 The serum levels of miR-126 from the controls, the Stage IV NSCLC patients, and the Stage I/II NSCLC patients were tested by stem-loop RT-PCR. There was no statistical difference between the controls and Stage I/II patients $(P>0.05)$, while there was statistical difference between the Stage I/II patients and Stage IV patients $(P<0.05)$

Hsa-miR-126 and hsa-miR-183 may serve as serum biomarkers for diagnosing metastatic NSCLC.

\section{Discussion}

Tumor metastasis is one of the most important prognostic factors for advanced NSCLC, which often results in treatment failure. So it seems urgent to detect metastasis in a convenient way at a relatively early stage.

It has been well established that miRNAs are present in human's peripheral blood. And some researches have focused on the sources of circulatory miRNAs. In healthy 


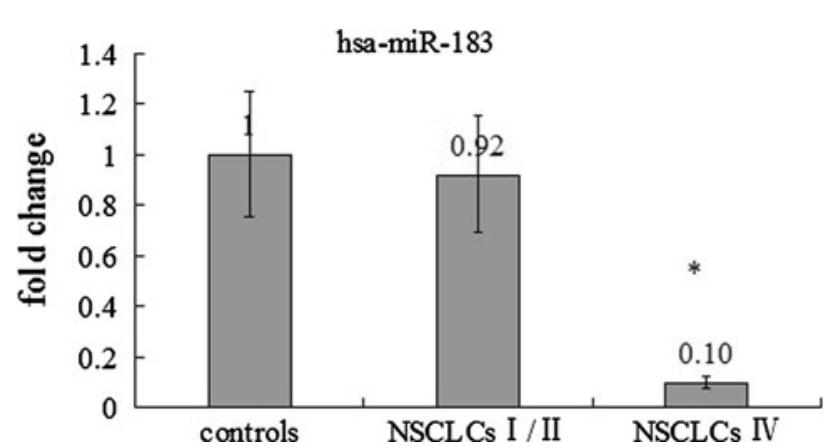

Fig. 5 The serum levels of miR-183 from the controls, the Stage IV NSCLC patients, and the Stage I/II NSCLC patients were tested by stem-loop RT-PCR. There was no statistical difference between the controls and Stage I/II patients $(P>0.05)$, while there was statistical difference between the Stage I/II patients and Stage IV patients $(P<0.05)$

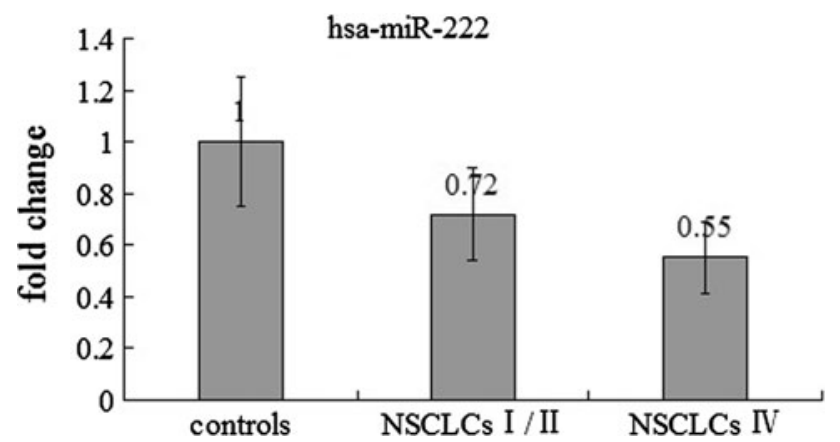

Fig. 6 The serum levels of miR-222 from the controls, the Stage IV NSCLC patients, and the Stage I/II NSCLC patients were tested by stem-loop RT-PCR. There was neither any statistical difference between the controls and Stage I/II patients $(P>0.05)$, nor between the Stage I/II patients and Stage IV patients $(P>0.05)$

individuals, by measuring miRNAs in the peripheral blood and blood cells, it was found that circulatory miRNAs came from blood cells. In cancer patients, many reports have clearly shown that cancer affect miRNA levels in the bloodstream, although it is still unclear how tumor miRNAs make their way into the bloodstream. So far, there has been no definitive conclusion of cancer-related miRNAs sources, but it is for certain that they have no relation with blood cells (Chen et al. 2008). However, there are mainly two conjectures about the sources of cancer-related miRNAs. They may originate from cancer cells in the circulation (Mitchell et al. 2008). They may be present as a result of tumor cells dying and getting lysed or tumor cells releasing miRNAs into the surrounding environment (Chin and Slack 2008). The other conjecture is that they may come from tumor-derived exosomes. Some researchers detected certain miRNA levels of cancer cells and circulating tumor exosomes from ovarian cancer patients by using a modified MACS procedure with anti-EpCAM, and it was proposed that cancer-related miRNAs in the circulation came from tumor-derived exosomes (Taylor and Gercel-Taylor 2010).

Quantification of miRNAs by stem-loop RT-PCR and high-flux sequencing technology were used to confirm that the vast majorities of the small RNAs were miRNAs in peripheral blood (Chen et al. 2008). Other qualitative, quantitative, or semiquantitative experiments also indicated that miRNAs exist in human's peripheral blood (Lawrie et al. 2008; Resnick et al. 2009; Chim et al. 2008; Gilad et al. 2008). In fact, stem-loop RT primers are better than conventional ones in terms of RT efficiency and specificity. Furthermore, they are not affected by genomic DNA contamination. Precise quantification is achieved routinely with as little as $25 \mathrm{pg}$ of total RNA for most miRNAs. This method enables fast, accurate, and sensitive miRNA expression profiling and can identify and monitor potential biomarkers specific to tissues or diseases (Chen et al. 2005). This present study applied the stem-loop RT-PCR method to show that certain miRNAs such as miR-125a-5p, miR-126, miR-183, miR-200, miR-221, and miR-222 were present in peripheral blood. At the same time, the serum samples can be obtained atraumatically and conveniently.

Besides, miRNAs are stable in peripheral blood. Given that serum has been reported to contain high levels of RNase activity, miRNAs exist in a form that is resistant to serum nuclease activity. Studies have shown that the levels of endogenous miRNAs remain stable in many other harsh conditions such as prolonged room temperature incubation, repeated freeze-thawing procedures, boiling, superacid, and peralkaline. The intrinsic stability of miRNAs may attribute to their small size and chemical structure, but not the extrinsic conditions (Mitchell et al. 2008; Resnick et al. 2009; Chim et al. 2008).

In addition, miRNA measurements are substantially identical in serum compared with in plasma, indicating that both serum and plasma samples will be suitable for the investigations of miRNAs as blood-based biomarkers. Studies also indicate that there is not any significant difference of miRNAs level between men and women (Chen et al. 2008; Mitchell et al. 2008). Our study demonstrated that the serum levels for miR-125a-5p, miR-126, miR-183, miR-200, miR-221, and miR-222 between the male and female controls were not significantly different, which kept identical with the previous studies.

Four major hallmarks-unlimited cell proliferation, autonomous growth, resistance to growth inhibitory signals, and escape from apoptosis-are central to the transformation of a normal cell to a cancer phenotype, the initial step of tumorigenesis. MiRNAs have recently been implicated as important modulators of these cell growth and survival processes, and their deregulation can initiate malignant transformation and unchecked cellular growth (Du and Pertsemlidis 2010). The regulatory mechanisms of 
miRNA expression includes various ways such as epigenetic changes (methylation for example), transcription factor misadjustment that controls the expression of miRNA coding gene, chromosome abnormality, genic mutation, single-nucleotide polymorphism, mechanism disorders of miRNA biosynthesis, and so on ( $\mathrm{Du}$ and Pertsemlidis 2010). And as continuously being shown, miRNAs can act as both oncogenes and tumor suppressors (Esquela-Kerscher and Slack 2006). Through various techniques, numerous groups have shown that different cancer types including NSCLC have distinct miRNA profiles. This may explain why some cancer-related miRNAs are up-regulated and others are down-regulated.

In our present study, all of the six specified miRNAs have been reported to be involved in lung cancer metastases in vitro; however, the serum level for some of them was of no significant difference between the healthy controls and the patients. Only hsa-miR-126, hsa-miR-183, and hsa-miR-222 showed significant difference between the serum of the Stage IV NSCLC patients and the controls. And the expression levels for these three miRNAs were lower in the patients than in the controls. It seems difficult to understand the relation and function of tissue and serum miRNAs levels.

As described above, there is a hypothesis that tumor cells use exosomes/microvesicles to transport genetic information, including miRNAs, to surrounding cells and thereby support tumor growth and progression (Skog et al. 2008). Moreover, circulating miRNAs might modulate immune responses (Valenti et al. 2007; Baj-Krzyworzeka et al. 2007): For example, micro vesicles derived from human melanoma and colon cancer can promote tumor growth and immune escape by mediating the differentiation of monocytes toward TGF $\beta$-secreting myeloid suppressive cells (Valenti et al. 2007). However, it has not been investigated if these effects are mediated by the miRNAs contained in the microvesicles.

There are also contradictive assumptions about the question. Wang et al. (2009b) concluded from their study that specific miRNAs are released into the blood stream after drug induced liver injury leading to a down-regulation in the tissue. In contrast to that, Tanaka et al. 2009 speculated that tumor cells rely on the specific intake of miRNAs from circulating micro vesicles. These different viewpoints clearly demonstrate a need for additional studies to elucidate the relation of tissue and serum miRNAs expression levels.

In fact, many researches have indicated that miRNAs play essential roles in tissue differentiation during normal development and tumorigenesis (Mascaux et al. 2009). Certain miRNAs are differentially expressed in lung squamous cell carcinomas and adenocarcinomas. A group of these miRNAs shows considerable tissue specificity (Lu et al. 2005). For example, miR-205 is believed to be a marker of stratified squamous epithelium (Ryan et al. 2006), and its expression is retained in squamous cell cancers of the upper and lower respiratory tracts (Yanaihara et al. 2006; Jiang et al. 2005). Actually, the differential expression of miR-205 in lung squamous cell cancers but not in adenocarcinomas provides a rationale for the classification of NSCLCs based on miRNA expression profiling. Quantification of miR-205 level has recently been optimized for formalin-fixed and paraffin-embedded samples, so that levels of miR-205 can now be used to classify NSCLCs with a high degree of sensitivity and specificity (Lebanony et al. 2009). It was found that it had $100 \%$ sensitivity and $100 \%$ specificity in differentiating squamous cell cancers and adenocarcinomas for the lung cancer resections by applying quantitative reverse transcription-PCR to analyze the hsa-miR-205 and hsa-miR-21 expression together (Bishop et al. 2010). However, so far there have been no data about the serum level of miR-205 in NSCLCs. Our study demonstrated that there was no significant difference in the serum levels for certain miRNAs including miR-125a-5p, miR-126, miR-183, miR-200, miR-221, and miR-222 between the lung squamous cell cancer patients and adenocarcinoma patients, indicating that the serum levels of these miRNAs may have no relation to the pathological types of NSCLCs.

As for the serum levels for the six cited miRNAs, our study showed significant difference for hsa-miR-126, hsamiR-183, and hsa-miR-222 between the Stage IV NSCLC patients and the controls. Further study showed that there was no statistical difference for hsa-miR-126, hsa-miR183, and hsa-miR-222 between the controls and Stage I/II patients, which demonstrated that the expression of hsamiR-126, hsa-miR-183, and hsa-miR-222 kept stable at the early stage of NSCLC. Furthermore, it showed statistical difference for hsa-miR-126 and hsa-miR-183 between the Stage I/II patients and Stage IV patients, but not for hsamiR-222, which indicated that the serum levels for hsamiR-126 and hsa-miR-183 changed, but hsa-miR-222 kept at a stable level as the metastases developed. So we concluded that the levels of hsa-miR-126 and hsa-miR-183 were closely related to lung cancer metastases and these two miRNAs had the potential to serve as serum biomarkers for diagnosing NSCLC metastases. Interestingly, our study demonstrated that the three miRNAs - hsa-miR126, hsa-miR-183, and hsa-miR-222 - were all downregulated in the patients' serum, which indicated that they could inhibit cancer progression, while hsa-miR-126 and hsa-miR-183 could play an important role in inhibiting lung cancer metastases.

The data of this study showed that the serum level for miR-126 was 2.5-fold decrease in the Stage IV NSCLC patients versus the controls. By analyzing a miRNA profile 
of SCC samples of Chinese lung cancer patients, Yang et al. (2010) identified that miR-126 was one of the miRNAs that were down-regulated in the tumors compared with non-cancerous lung tissues. The over-expression of miR-126 results in a decrease in Crk protein, which is a member of a family of adaptor proteins involved in intracellular signal pathways altering cell adhesion, proliferation, and migration. As increased expression of Crk has been described in lung cancer and associated with increased tumor invasiveness, so miR-126 alters lung cancer cell phenotype by inhibiting adhesion, migration, and invasion, and the effects on invasion may be partially mediated through Crk regulation (Crawford et al. 2008).

With regard to hsa-miR-183, our data showed that the level was up to tenfold decrease in the Stage IV NSCLC patients versus the controls. It was identified that several potential downstream effectors including ezrin and more than 30 genes were involved in metastases in pulmonary giant cell cancer (Wang et al. 2008). Further study showed that ezrin and parathyroid hormone-related protein were up-regulated at sites of bone metastasis as detected by RTPCR and immunohistochemistry in HARA lung squamous carcinoma cells, which offers new mechanistic insights into the metastasis of lung cancer and provides potential targets for the prevention and treatment of lung cancer metastasis (Deng et al. 2007).

In our study, the serum level for miR-222 was nearly twofold decrease in the Stage IV NSCLC patients versus the controls with significant difference. However, the serum level for miR-221 between the two groups showed no significant difference. Some previous studies proved that miR-221 \& 222 function in the same way. MiR-221 \& 222 induce TRAIL resistance and enhance cellular migration through the activation of the AKT pathway and metallopeptidases by targeting PTEN and TIMP3 tumor suppressors. And the MET oncogene is involved in miR222 and miR-221 activation through the c-Jun transcription factor (Garofalo et al. 2009). Our study showed that miR222 was at a relatively higher level, indicating that examination in the serum may be less sensitive or perhaps only a part of microRNAs in the tumor cells can be released to the serum. In this way, it is easy to understand that the serum levels for other three miRNAs miR-125a-5p, miR-200, and miR-221 were of no significant difference between the patients and the controls.

For patients with suspected metastatic lung cancer, histopathological diagnosis is the gold standard; however, sometimes it is difficult to obtain tissue for diagnosis. Computed tomography (CT) even combined with positron emission tomography (PET) is usually used to detect the metastasis or stage the disease. Even though it is a widely used tool for diagnosing and managing suspected lung cancer, it has limited sensitivity and specificity, besides
PET is very expensive. In contrast, detecting the serum level of certain miRNAs by stem-loop RT-PCR is relatively convenient and economical. Additionally, it may be applied to improve the accuracy for diagnosing metastatic lung cancer combined with other currently widely used tools.

Although currently there are some problems with using miRNAs as cancer biomarkers such as the unclearly cut reference range and the lack of large-sample data, our study extends the potential of hsa-miR-126 and hsa-miR183 to serve as molecular markers for human primary lung cancer. The serum levels for these miRNAs could be identified as an indicator for metastatic NSCLCs. And they may be applied clinically combining with other lung cancer-specific miRNAs such as miR-21, miR-210, and miR486-5p in the future (Shen et al. 2011). Since there have been some meaningful findings, we are planning to further study these miRNAs in NSCLCs.

Acknowledgments The study was supported by Jiangsu Provincial Scientific Committee and Health Department and Kejiaoxingwei Project (KF200939).

Conflict of interest We declare that we have no conflict of interest.

Open Access This article is distributed under the terms of the Creative Commons Attribution Noncommercial License which permits any noncommercial use, distribution, and reproduction in any medium, provided the original author(s) and source are credited.

\section{References}

Baj-Krzyworzeka M, Szatanek R, Weglarczyk K, Baran J, Zembala M (2007) Tumour-derived microvesicles modulate biological activity of human monocytes. Immunol Lett 113:76-82. doi: 10.1016/j.imlet.2007.07.014

Bishop JA, Benjamin H, Cholakh H, Chajut A, Clark DP, Westra WH (2010) Accurate classification of non-small cell lung carcinoma using a novel microRNA-based approach. Clin Cancer Res 16:610. doi:10.1158/1078-0432.CCR-09-2638

Chen C, Ridzon DA, Broomer AJ, Zhou Z, Lee DH et al (2005) Realtime quantification of microRNAs by stem-loop RT-PCR. Nucleic Acids Res 33:e179. doi:10.1093/nar/gni178

Chen X, Ba Y, Ma L et al (2008) Characterization of microRNAs in serum: a novel class of biomarkers for diagnosis of cancer and other diseases[J]. Cell Res 18(10):997-1006. doi:10.1038/cr. 2008.282

Chim SS, Shing TK, Hung EC et al (2008) Detection and characterization of placental microRNAs in maternal plasma. Clin Chem 54(3):482-490. doi:10.1373/clinchem.2007.097972

Chin LJ, Slack FJ (2008) A truth serum for cancer-microRNAs have major potential as cancer biomarkers. Cell Res 18:983-984. doi: 10.1038/cr.2008.290

Coello MC, Luketich JD, Litle VR (2004) Prognostic significance of micrometastasis in non-small-cell lung cancer. Clin Lung Cancer 5(4):214-225 PMID: 14967073

Crawford M, Brawner E, Batte K, Yu L, Hunter MG, Otterson GA, Nuovo G, Marsh CB, Nana-Sinkam SP (2008) MicroRNA-126 inhibits invasion in non-small cell lung carcinoma cell lines. Biochem Biophys Res Commun 373(4):607-612. doi:10.1016/ j.bbrc.2008.06.090 
Deng X, Tannehill-Gregg SH, Nadella MV et al (2007) Parathyroid hormone-related protein and ezrin are up-regulated in human lung ccncer bone metastases. Clin Exp Metastasis 24(2):107-119. doi:10.1007/s10585-007-9059-9

Du L, Pertsemlidis A (2010) MicroRNAs and lung cancer: tumors and 22-mers. Cancer Metastasis Rev 29:109-122. doi:10.1007/ s10555-010-9204-9

Dutt A, Ramos AH, Hammerman PS, Mermel C, Cho J, Sharifnia T et al (2011) Inhibitor-sensitive FGFR1 amplification in human non small cell lung cancer. PLoS One 6(6):e20351. doi:10.1371/ journal.pone.0020351

Esquela-Kerscher A, Slack FJ (2006) Oncomirs-microRNAs with a role in cancer. Nat Rev Cancer 6:259-269. doi:10.1038/nrc1840

Garofalo M, Di Leva G, Romano G, Nuovo G, Suh SS, Ngankeu A et al (2009) MiR-221 \& 222 regulate TRAIL resistance and enhance tumorigenicity through PTEN and TIMP3 downregulation. Cancer Cell 16(6):498-509. doi:10.1016/j.ccr.2009.10.014

Gilad S, Meiri E, Yogev Y et al (2008) Serum microRNAs are promising novel biomarkers. PLoS One 3(9):e3148. doi: 10.1371/journal.pone.0003148

Jemal A, Siegel R, Ward E, Murray T, Xu J, Smigal C, Thun MJ (2006) Cancer statistics. CA Cancer J Clin 56:106-130. doi: 10.3322/canjclin.56.2.106

Jiang J, Lee EJ, Gusev Y, Schmittgen TD (2005) Real-time expression profiling of microRNA precursors in human cancer cell lines. Nucleic Acids Res 33:5394-5403. doi:10.1093/ nar/gki863

Lawrie CH, Gal S, Dunlop HM et al (2008) Detection of elevated levels of tumour-associated microRNAs in serum of patients with diffuse large B-cell lymphoma[J]. $\mathrm{Br} \mathrm{J}$ Haematol 141(5):672-675. doi:10.1111/j.1365-2141.2008.07077.x

Lebanony D, Benjamin H, Gilad S et al (2009) Diagnostic assay based on hsa-miR-205 expression distinguishes squamous from nonsquamous non-small-cell lung carcinoma. J Clin Oncol 27:2030-2037. doi:10.1200/JCO.2008.19.4134

Lu J, Getz G, Miska EA, Alvarez-Saavedra E, Lamb J, Peck D et al (2005) MicroRNA expression profiles classify human cancers. Nature 435(7043):834-838. doi:10.1038/nature03702

Mascaux C, Laes JF, Anthoine G et al (2009) Evolution of microRNA expression during human bronchial squamous carcinogenesis. Eur Respir J 33:352-359. doi:10.1183/09031936.00084108

Mitchell PS, Parkin RK, Kroh EM et al (2008) Circulating microRNAs as stable blood-based markers for cancer detection[J]. Proc Natl Acad Sci USA 105(30):10513-10518. doi: 10.1073/pnas.0804549105

Resnick KE, Alder H, Hagan JP, Richardson DL, Croce CM, Cohn DE (2009) The detection of differentially expressed microRNAs from the serum of ovarian cancer patients using a novel realtime
PCR platform[J]. Gynecol Oncol 112(1):55-59. doi: 10.1016/j.ygyno.2008.08.036

Roybal JD, Zang Y, Ahn YH et al. (2011) MiR-200 inhibits lung adenocarcinoma cell invasion and metastasis by targeting Flt1/ VEGFR1. Mol Cancer Res 9(1):25-35. doi:10.1158/1541-7786. [Epub 2010 Nov 29]

Ryan DG, Oliveira-Fernandes M, Lavker RM (2006) MicroRNAs of the mammalian eye display distinct and overlapping tissue specificity. Mol Vis 12:1175-1184

Shen J, Todd NW, Zhang H, Yu L, Lingxiao X, Mei Y et al (2011) Plasma microRNAs as potential biomarkers for non-small-cell lung cancer. Lab Invest 91:579-587. doi:10.1038/labinvest. 2010.194

Skog J, Wurdinger T, van Rijn S, Meijer DH, Gainche L, SenaEsteves $\mathrm{M}$ et al (2008) Glioblastoma microvesicles transport RNA and proteins that promote tumour growth and provide diagnostic biomarkers. Nat Cell Biol 10:1470-1476. doi: $10.1038 /$ ncb1800

Tanaka M, Oikawa K, Takanashi M, Kudo M, Ohyashiki J, Ohyashiki K, Kuroda M (2009) Down-regulation of miR-92 in human plasma is a novel marker for acute leukemia patients. PLoS One 4:e5532. doi:10.1371/journal.pone.0005532

Taylor DD, Gercel-Taylor C (2010) MicroRNA signatures of tumorderived exosomes as diagnostic biomarkers of ovarian cancer. Gynecol Oncol 110(1):13-21. doi:10.1016/j.ygyno.2008.04.033

Valenti R, Huber V, Iero M, Filipazzi P, Parmiani G, Rivoltini L (2007) Tumor-released microvesicles as vehicles of immunosuppression. Cancer Res 67:2912-2915. doi:10.1158/0008-5472. CAN-07-0520

Wang G, Mao W, Zheng S (2008) MicroRNA-183 regulates Ezrin expression in lung cancer cells. FEBS Lett 582:3663-3668. doi: 10.1016/j.febslet.2008.09.051

Wang G, Mao W, Zheng S, Ye J (2009a) Epidermal growth factor receptor-regulated miR-125a-5p-a metastatic inhibitor of lung cancer. FEBS J 276(19):5571-5578. doi:10.1111/j.1742-4658. 2009.07238.x

Wang K, Zhang S, Marzolf B, Troisch P, Brightman A, Hu Z, Hood LE, Galas DJ (2009b) Circulating microRNAs, potential biomarkers for drug-induced liver injury. Proc Natl Acad Sci USA 106:4402-4407. doi:10.1073/pnas.0813371106

Yanaihara N, Caplen N, Bowman E et al (2006) Unique microRNA molecular profiles in lung cancer diagnosis and prognosis. Cancer Cell 9:189-198. doi:10.1016/j.ccr.2006.01.025

Yang Y, Li X, Yang Q, Wang X, Zhou Y, Jiang T et al (2010) The role of microRNA in human lung squamous cell carcinoma. Cancer Genet Cytogenet 200(2):127-133. doi:10.1016/ j.cancergencyto.2010.03.014 